Author affiliations and support information (if applicable) appear at the end of this article.

Published at ascopubs.org/journal/jco on December 27, 2016.

M.J.R. and H.S.v.M. contributed equally to this work.

Clinical trial information: NTR2644.

Corresponding author: Marrije R. Buist, MD, Department of Gynecologic Oncology, Academic Medical Center, Center of Gynecologic Oncology, PO Box 22660, 1100 DD Amsterdam, the Netherlands; e-mail: m.r.buist@amc.nl.

C 2016 by American Society of Clinical Oncology

0732-183X/17/3506w-613w/\$20.00

\title{
Laparoscopy to Predict the Result of Primary Cytoreductive Surgery in Patients With Advanced Ovarian Cancer: A Randomized Controlled Trial
}

Marianne J. Rutten, Hannah S. van Meurs, Roelien van de Vrie, Katja N. Gaarenstroom, Christiana A. Naaktgeboren, Toon van Gorp, Henk G. Ter Brugge, Ward Hofhuis, Henk W.R. Schreuder, Henriette J.G. Arts, Petra L.M. Zusterzeel, Johanna M.A. Pijnenborg, Maarten van Haaften, Guus Fons, Mirjam J.A. Engelen, Erik A. Boss, M. Caroline Vos, Kees G. Gerestein, Eltjo M.J. Schutter, Brent C. Opmeer, Anje M. Spijkerboer, Patrick M.M. Bossuyt, Ben Willem Mol, Gemma G. Kenter, and Marrije R. Buist

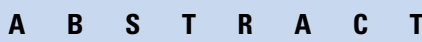

\section{Purpose}

To investigate whether initial diagnostic laparoscopy can prevent futile primary cytoreductive surgery (PCS) by identifying patients with advanced-stage ovarian cancer in whom $>1 \mathrm{~cm}$ of residual disease will be left after PCS.

\section{Patients and Methods}

This multicenter, randomized controlled trial was undertaken within eight gynecologic cancer centers in the Netherlands. Patients with suspected advanced-stage ovarian cancer who qualified for PCS were eligible. Participating patients were randomly assigned to either laparoscopy or PCS. Laparoscopy was used to guide selection of primary treatment: either primary surgery or neoadjuvant chemotherapy followed by interval surgery. The primary outcome was futile laparotomy, defined as a PCS with residual disease of $>1 \mathrm{~cm}$. Primary analyses were performed according to the intention-to-treat principle.

\section{Results}

Between May 2011 and February 2015, 201 participants were included, of whom 102 were assigned to diagnostic laparoscopy and 99 to primary surgery. In the laparoscopy group, 63 (62\%) of 102 patients underwent PCS versus 93 (94\%) of 99 patients in the primary surgery group. Futile laparotomy occurred in 10 (10\%) of 102 patients in the laparoscopy group versus 39 (39\%) of 99 patients in the primary surgery group (relative risk, $0.25 ; 95 \% \mathrm{Cl}, 0.13$ to $0.47 ; P<.001$ ). In the laparoscopy group, three (3\%) of 102 patients underwent both primary and interval surgery compared with $28(28 \%)$ of 99 patients in the primary surgery group $(P<.001)$.

\section{Conclusion}

Diagnostic laparoscopy reduced the number of futile laparotomies in patients with suspected advanced-stage ovarian cancer. In women with a plan for PCS, these data suggest that performance of diagnostic laparoscopy first is reasonable and that if cytoreduction to $<1 \mathrm{~cm}$ of residual disease seems feasible, to proceed with PCS.

\section{J Clin Oncol 35:613-621. (c) 2016 by American Society of Clinical Oncology}

\section{INTRODUCTION}

Epithelial ovarian cancer is the seventh most common cancer in women worldwide, and the 5-year survival rate ranges from $30 \%$ to $50 \%{ }^{1}$ Standard treatment consists of primary cytoreductive surgery (PCS) followed by platinumbased chemotherapy. ${ }^{2,3}$ Currently, discussion on primary treatment of patients with advancedstage ovarian cancer who should undergo PCS or interval cytoreductive surgery is ongoing. ${ }^{4}$ Two randomized trials showed the noninferiority of interval cytoreductive surgery in patients with International Federation of Gynecology and Obstetrics (FIGO) stage IIIC to IV. ${ }^{5,6}$ However, a post hoc analysis of this study showed that prognosis was favorable in patients with metastases $<45 \mathrm{~mm}$ and stage IIIC disease who undergo PCS. In contrast, patients with larger metastases and stage IV disease benefited more from interval cytoreductive surgery. ${ }^{7}$ Recently, 
another single-institution analysis showed that the best survival outcomes were observed in patients deemed eligible for PCS. ${ }^{8}$ Survival is strongly correlated with the size of residual tumor; therefore, the aim of cytoreductive surgery is to leave no residual tumor or at least a maximum diameter of $<1 \mathrm{~cm} .{ }^{9-11}$ The dilemma is that PCS that results in no residual disease may require extensive surgery with a subsequent higher risk of morbidity. ${ }^{5}$ If at PCS extensive disease is present, surgery could be ceased, and neoadjuvant chemotherapy (NACT) with interval surgery could be a good alternative treatment. ${ }^{4}$ Therefore, the identification of patients with extensive disease who are likely to have $>1 \mathrm{~cm}$ of residual tumor after PCS, defined as a futile laparotomy, is important. ${ }^{12}$ Current, noninvasive diagnostic methods such as physical examination, ultrasonography, abdominal computed tomography (CT), and serum tumor markers like CA125 and carcinoembryonic antigen fail to predict completeness of surgery accurately. Although numerous investigators have tried to create prediction models that are based on various imaging techniques and clinical features, none of these models have proven to be useful to prevent futile laparotomy in daily practice. $^{13}$

On the basis of previous studies, we hypothesized that laparoscopy can reduce the number of futile laparotomies by predicting the outcome of PCS without increasing the risk of omitting primary surgery to patients with resectable disease or increasing the risk for complications. ${ }^{14-16}$ To investigate this hypothesis, we conducted a multicenter, randomized controlled trial that used laparoscopy as a diagnostic tool to guide choice of primary treatment in patients with suspected advanced-stage epithelial ovarian cancer.

\section{PATIENTS AND METHODS}

\section{Study Design and Participants}

This randomized controlled trial was performed within eight gynecologic cancer centers in the Netherlands. Patients between 18 and 80 years of age with suspected advanced-stage ovarian cancer (FIGO stage IIB or higher ${ }^{17}$ ) were eligible whenever PCS seemed possible after conventional work-up. Conventional work-up consisted of medical history, complete physical and gynecologic examination, measurement of serum tumor markers (CA125 and carcinoembryonic antigen), calculation of the Risk of Malignancy Index score, ${ }^{18}$ sonography of the pelvic area, CT scan of the abdomen, and imaging of the thorax (CT thorax preferred). Biopsy was not obligatory in the conventional work-up because high suspicion of advanced-stage ovarian cancer was already the reason for surgical intervention. However, the presence of advanced-stage ovarian cancer was always histologically confirmed before the start of NACT.

Exclusion criteria were a World Health Organization performance status $\geq 3$ or any contraindications for laparoscopy. Other exclusion criteria were presence of a large immobile pelvic tumor; imaging evidence of the presence of intrahepatic metastatic disease of $>1 \mathrm{~cm}$; extraabdominal metastatic disease (not to exclude patients with only inguinal lymph nodes or pleural fluid); periaortic lymphadenopathy $>1 \mathrm{~cm}$ above the level of the renal veins; extensive peritoneal carcinomatosis at the diaphragmatic level; and extensive bowel mesentery involvement, which suggests that cytoreductive surgery that would result in $<1 \mathrm{~cm}$ of residual disease seemed not feasible or would increase the risk of major complications. In the presence of these signs of extensive disease, the patient was considered not eligible for PCS and thus not randomly assigned in the current study. ${ }^{6}$ The decision about eligibility for PCS was made at a multidisciplinary session attended by a gynecologic oncologist, medical oncologist, pathologist, and radiologist.
Ethical approval was obtained from the medical ethics committee of the Academic Medical Center of Amsterdam (MEC10/183). All patients were treated in a gynecologic oncology center, and laparoscopies and cytoreductive surgeries were performed by an accredited gynecologic oncologist. Standard chemotherapy comprised six cycles of carboplatin and paclitaxel $175 \mathrm{mg} / \mathrm{m}^{2}$ every 3 weeks.

\section{Random Assignment and Masking}

After written informed consent, participants were randomly assigned (1:1) to either diagnostic laparoscopy (intervention arm) or to PCS followed by chemotherapy (standard arm). Random assignment was performed centrally with a Web-based tool that used a permuted-block design and stratification by gynecologic cancer center. Participants and investigators were not masked to treatment allocation.

\section{Procedure}

In the intervention arm, diagnostic laparoscopy was performed within 3 weeks after random assignment. An open laparoscopy was performed to inspect the whole abdomen systematically. The decision that cytoreduction to $<1 \mathrm{~cm}$ of residual disease was not feasible was made by the gynecologic oncologist on the basis of the following laparoscopic findings: extensive agglutinated intra-abdominal metastatic disease (including spleen or retrohepatic area involvement), extensive serosa invasion of the intestines and/or mesenterial deposits (which would make multiple bowel resections of $>1.5 \mathrm{~m}$ of bowel necessary to reach complete cytoreductive surgery), and extensive (irresectable) peritoneal metastases at the diaphragmatic level. If PCS that would leave at least $<1 \mathrm{~cm}$ was considered feasible, PCS was performed and followed by six cycles of chemotherapy. If PCS with maximal effort to remove all tumor to $<1 \mathrm{~cm}$ of disease was considered not feasible at laparoscopy, patients were primarily treated with three courses of NACT followed by interval cytoreductive surgery. In the standard arm, all patients were assigned to PCS. The goal of surgery in all cases was to resect all macroscopic tumors. In both study arms, a second laparotomy was considered after three cycles of chemotherapy in case $>1 \mathrm{~cm}$ of residual tumor was left after the first surgical attempt. The amount and localization of residual disease was reported on a standard form by the gynecologic oncologist. Follow-up consisted of visits every 3 months for the first 2 years followed by visits every 6 months up to 5 years. Recurrent disease was reported if patients presented with clinical symptoms and laboratory results or image evidence showed recurrent disease.

\section{Outcomes}

The primary outcome measure of the study was a futile laparotomy, defined as residual tumor with a maximum diameter of $>1 \mathrm{~cm}$ after PCS. The aim of this study was to guide treatment decisions for either PCS or interval cytoreductive surgery, to reduce the number of patients subjected to multiple laparotomies, and to select patients suitable for PCS. Secondary outcomes were adverse events and progression-free and overall survival. Adverse events were graded by the Common Terminology Criteria for Adverse Events (version 4.0) and assessed during treatment and at each clinical visit.

\section{Statistical Analysis}

Analyses were performed on an intention-to-treat basis. We calculated the proportion of patients with futile laparotomies in each arm. We tested for differences with the $\chi^{2}$ test, or if a group contained fewer than five patients, the Fisher's exact test, with a two-sided significance level of .05.

In the survival analyses, survival time was considered censored at the time patients were last known to be alive or at the end of follow-up. Survival was compared by estimating Kaplan-Meier curves in each arm and tested by using the log-rank test. Hazard ratios were calculated with a Cox proportional hazards model. Progression-free survival was defined as time from random assignment until the date of clinical progression, recurrence, or death, whichever came first. 
The rate of incomplete PCS (ie, leaving $>1 \mathrm{~cm}$ of residual disease) after conventional work-up in the Netherlands was estimated to be $40 \%{ }^{10,19}$ We hypothesized that laparoscopy would reduce this rate to $20 \%$. To achieve a power of $80 \%$, 90 participants per arm had to be included. With a consideration of $10 \%$ possible attrition, the trial sample size was set at 200 patients.

Statistical analysis was performed with SPSS version 23.0 software (IBM Corporation, Chicago, IL). This study was registered in the Netherlands Trial Register (NTR2644), and the study protocol has been published. ${ }^{20}$

\section{RESULTS}

\section{Patients}

Between May 2011 and February 2015, we enrolled 202 patients. One patient was randomly assigned incorrectly because there was no suspicion of advanced-stage ovarian cancer; this patient was therefore excluded from all analyses. Data on the remaining 201 patients were included in the intention-to-treat analysis; 102 were assigned to receive laparoscopy before surgery and 99 to PCS (Fig 1). All patients received a preoperative CT scan of the abdomen and lower thorax and a chest x-ray or CT scan of the thorax. The baseline characteristics were well balanced between the two treatment groups (Table 1).

Definitive histologic diagnosis, FIGO stage, and histologic grade by Silverberg ${ }^{21}$ were obtained after surgery (Table 2). The final pathology showed adenocarcinoma of the ovary, tubes, or peritoneum in 174 (87\%) of the 201 patients, with serous adenocarcinoma in the majority of cases. Ten patients (5\%) had other malignancies. Fourteen patients $(7 \%)$ had benign disease, equally divided between the groups. Among the 174 patients with ovarian cancer, $80 \%$ had FIGO stage IIIC or IV disease, equally divided between the groups.

In the laparoscopy group, 63 (62\%) of 102 patients underwent PCS after laparoscopy; 39 (38\%) of 102 patients were assigned to NACT followed by interval surgery. Reasons at laparoscopy to withhold from PCS were mostly because of extensive agglutinated intra-abdominal metastatic disease and extensive peritoneal metastases at the diaphragmatic level. In four of these patients, no

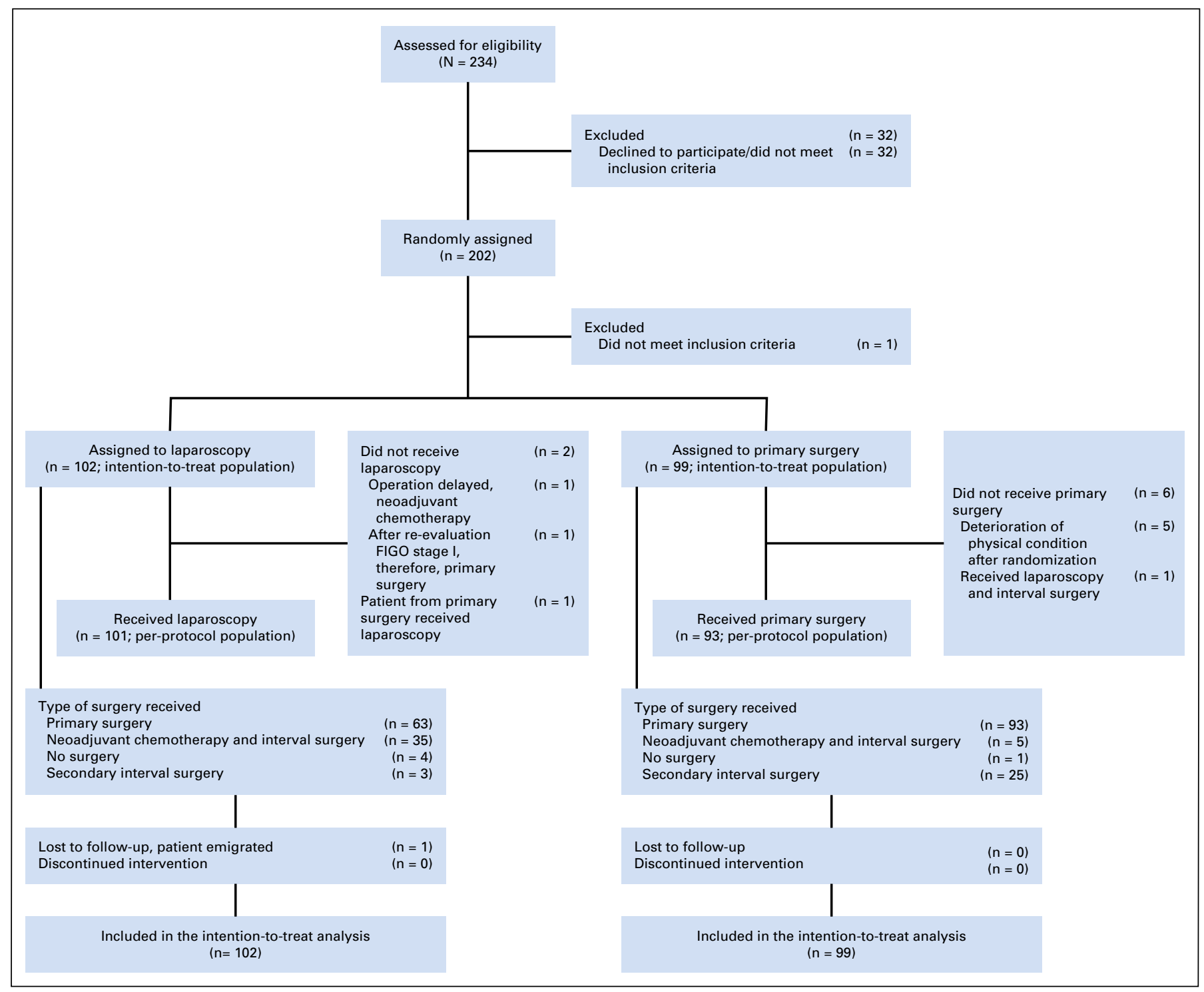

Fig 1. CONSORT diagram. FIGO, International Federation of Gynecology and Obstetrics. 


\begin{tabular}{|c|c|c|}
\hline Characteristic & Laparoscopy Before Surgery, No. (\%) & Primary Surgery, No. (\%) \\
\hline No. of patients & 102 & 99 \\
\hline Mean age (SD), years & 64 (8.9) & $64(9.8)$ \\
\hline Mean BMI (SD), $\mathrm{kg} / \mathrm{m}^{2}$ & $26.5(5.4)$ & $25.7(3.9)$ \\
\hline Postmenopausal & $91(90)$ & $86(87)$ \\
\hline Median Risk of Malignancy Index (IOR) & $3,942(1,521-10,611)$ & $3,486(1,467-9,990)$ \\
\hline \multicolumn{3}{|l|}{ WHO performance status } \\
\hline 0 & 70 (69) & $61(62)$ \\
\hline 1 & $28(28)$ & $30(30)$ \\
\hline 2 & $4(4)$ & $8(8)$ \\
\hline Median CA125 before treatment (IQR), kU/L & $655(211-1,440)$ & $604(246-1,446)$ \\
\hline Median CEA before treatment (IQR), $\mu \mathrm{g} / \mathrm{L}$ & $1.60(1.0-3.2)$ & $1.60(1.0-3.5)$ \\
\hline Mean albumin before treatment (SD), g/dL & $38.0(6.9)$ & $40.7(5.9)$ \\
\hline
\end{tabular}

Abbreviations: BMI, body mass index; CEA, carcinoembryonic antigen; IQR, interquartile range; SD, standard deviation.

cytoreductive surgery was attempted because of progression while receiving chemotherapy or inoperability because of poor general condition. In the primary surgery group, 93 (94\%) of 99 patients underwent PCS. One patient erroneously received a diagnostic laparoscopy despite allocation to primary surgery; she was assigned to NACT. Five patients in the primary surgery group experienced a deterioration of their physical condition after random assignment and were considered unfit for PCS; they were assigned to NACT. Four of these patients received interval surgery (Fig 2).

\section{Primary Outcome}

Futile laparotomy that left $>1 \mathrm{~cm}$ of residual disease after primary surgery occurred in $10(10 \%)$ of 102 patients in the laparoscopy group versus 39 (39\%) of 99 patients in the primary surgery group (relative risk [RR], $0.25 ; 95 \% \mathrm{CI}, 0.13$ to $0.47 ; P<.001$ ). In the laparoscopy group, 27 (27\%) of 102 patients had any residual tumor $(>0 \mathrm{~cm})$ versus $56(57 \%)$ of 99 patients in the primary surgery group (RR, $0.47 ; 95 \%$ CI, 0.32 to $0.68 ; P<.001$; Table 3 ). The per-protocol analysis for the entire study group showed similar results (Appendix Table A1, online only). In the subgroup analysis of patients with confirmed stage IIIC or IV ovarian cancer, only six (8\%) of 71 patients in the laparoscopy group versus $32(46 \%)$ of 69 patients in the primary surgery group underwent a futile laparotomy (Table 3 ).

\section{Secondary Outcomes}

In the laparoscopy group, four (4\%) of 102 patients underwent both primary and interval laparotomy versus $28(28 \%)$ of 99 patients in the primary surgery group $(P<.001$; Table 3$)$.

\begin{tabular}{|c|c|c|}
\hline Characteristic & Laparoscopy Before Surgery, No. (\%) & Primary Surgery, No. (\%) \\
\hline No. of patients & 102 & 99 \\
\hline \multicolumn{3}{|l|}{ FIGO stage ${ }^{*}$} \\
\hline It & $5(6)$ & $4(4)$ \\
\hline$\|$ & $5(6)$ & $9(10)$ \\
\hline III & $66(76)$ & $70(78)$ \\
\hline IIIA & $0(0)$ & $0(0)$ \\
\hline IIIB & $6(7)$ & $8(9)$ \\
\hline IIIC & $60(69)$ & $62(69)$ \\
\hline IV & $11(13)$ & 7 (8) \\
\hline \multicolumn{3}{|l|}{ Histologic diagnosis } \\
\hline Benign & $7(7)$ & $7(7)$ \\
\hline Borderline & $2(2)$ & $1(1)$ \\
\hline Serous adenocarcinoma & $72(71)$ & $67(68)$ \\
\hline Mucinous adenocarcinoma & $1(1)$ & $7(7)$ \\
\hline Endometrioid adenocarcinoma & $4(4)$ & $4(4)$ \\
\hline Clear cell adenocarcinoma & $1(1)$ & $5(5)$ \\
\hline Mixed epithelial carcinoma & $2(2)$ & $0(0)$ \\
\hline Other malignancy & $8(8)$ & $2(2)$ \\
\hline Adenocarcinoma not further specified & $5(5)$ & $6(6)$ \\
\hline \multicolumn{3}{|l|}{ Tumor grade by Silverberg 21} \\
\hline 1 & $6(7)$ & $4(4)$ \\
\hline 2 & $11(12)$ & $15(17)$ \\
\hline 3 & $52(57)$ & $53(58)$ \\
\hline Unknown & $22(24)$ & $19(21)$ \\
\hline
\end{tabular}




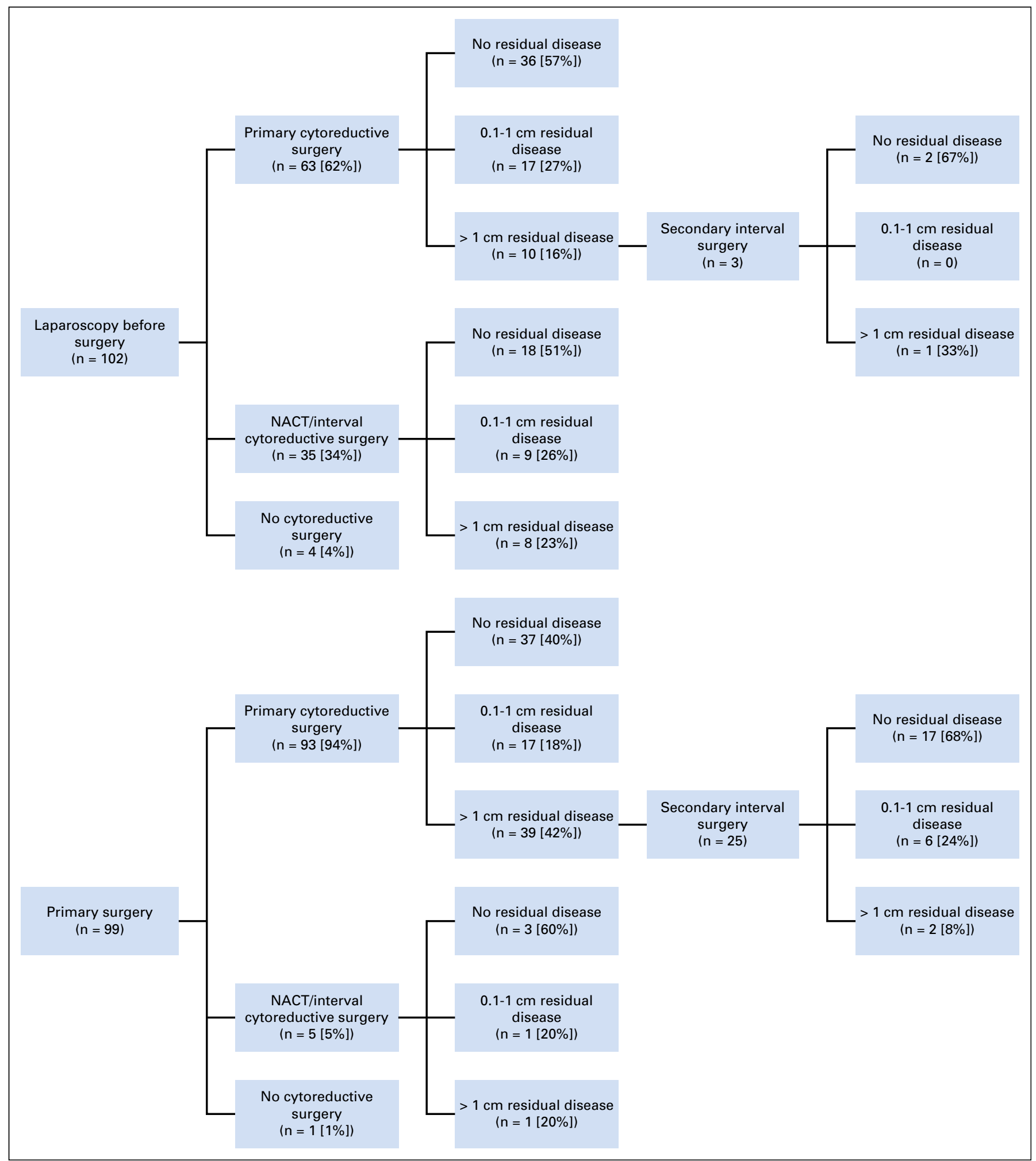

Fig 2. Residual disease after cytoreductive surgery in patients who had laparoscopy before surgery versus those who underwent primary surgery. NACT, neoadjuvant chemotherapy.

Reasons to omit extensive surgery at first laparotomy was if tumor resection to $<1 \mathrm{~cm}$ could only be reached by performing multiple bowel resections, extensive peritoneum stripping, or other extensive surgical procedures, which were presumed to give a higher complication rate. Nevertheless, extensive abdominal surgery was performed in $61(31 \%)$ of 196 patients, including peritoneal stripping, small or large bowel resection, artificial stoma construction, diaphragm stripping, splenectomy, and (partial) liver 


\begin{tabular}{|c|c|c|c|c|}
\hline Outcome & Laparoscopy Before Surgery, No. (\%) & Primary Surgery, No. (\%) & $\mathrm{RR}(95 \% \mathrm{Cl})$ & $P$ \\
\hline All patients & 102 & 99 & & \\
\hline Futile laparotomy any residual disease $\dagger$ & $27(27)$ & $56(57)$ & $0.47(0.32$ to 0.68$)$ & $<.001$ \\
\hline \multicolumn{5}{|l|}{ No. of laparotomies } \\
\hline 0 & $4(4)$ & $1(1)$ & & $<.001$ \\
\hline No. in FIGO stage IIIC or IV & 71 & 69 & & \\
\hline Futile laparotomy* & $6(8)$ & $32(46)$ & $0.18(0.08$ to 0.41$)$ & $<.001$ \\
\hline Futile laparotomy any residual disease $\dagger$ & $20(28)$ & $47(68)$ & $0.41(0.28$ to 0.62$)$ & $<.001$ \\
\hline \multicolumn{5}{|l|}{ No. of laparotomies in FIGO stage IIIC or IV } \\
\hline 0 & $3(4)$ & $1(1)$ & & $<.001$ \\
\hline 1 & $64(90)$ & $46(67)$ & & \\
\hline 2 & $4(6)$ & $22(32)$ & & \\
\hline
\end{tabular}

Abbreviations: FIGO, International Federation of Gynecology and Obstetrics; RR, relative risk.

$*>1 \mathrm{~cm}$ of residual disease after primary cytoreductive surgery.

$+>0 \mathrm{~cm}$ of residual disease after primary cytoreductive surgery.

resection in patients in whom complete cytoreductive surgery was feasible. No difference in extensive surgery between study groups was seen (Appendix Tables A2 and A3, online only).

In addition, analyses of the differences in the amount of residual disease per surgical strategy (primary or interval) were performed. No residual tumor was observed in 36 (57\%) of 63 patients who underwent PCS in the laparoscopy group versus 37 $(40 \%)$ of 93 patients in the primary surgery group $(P=.006$; Appendix Table A4, online only) and in 14 (41\%) of 34 patients and $16(25 \%)$ of 63 patients in the subgroup with stage IIIC or IV ovarian cancer, respectively (Appendix Table A5, online only). Complete remission after surgery and chemotherapy was seen in $65(66 \%)$ of 99 patients in the laparoscopy group versus $65(68 \%)$ of 95 in the primary surgery group (Appendix Table A2).

In the laparoscopy group, 22 patients had a grade 3 or 4 adverse event versus 26 in the primary surgery group (RR, 0.82; 95\% CI, 0.50 to $1.35 ; P=.44)$. Only one complication was related to laparoscopy: a wound infection that required antibiotics. Port-site metastases after laparoscopy were reported in three patients; these patients had stage IIIC or IV ovarian cancer (Appendix Table A6, online only). ${ }^{22-24}$

Median duration of follow-up was 34.6 months in the laparoscopy group and 33.8 months in the primary surgery group. At the last time of follow-up (April 8, 2016), 46 patients had died in the laparoscopy group and 42 in the primary surgery group (RR, 1.11 ; $95 \% \mathrm{CI}, 0.99$ to $1.25 ; P=.07$ ), which indicates comparable survival in both groups. Median progression-free survival was 13.7 months (interquartile range [IQR], 7.1 to 41.8 months) in the laparoscopy group and 15.2 months (IQR, 7.2 to $>34$ months) in the primary surgery group $(P=.88)$ with a hazard ratio of 0.97 (95\% CI, 0.69 to 1.38$)$. The median overall survival was 44.4 months (IQR, 16.8 to $>55$ months) in the laparoscopy group and 46.3 months (IQR, 13.9 to 52.6 months) in the primary surgery group $(P=.94)$ with a hazard ratio of 1.33 (95\% CI, 0.89 to 1.98; Appendix Table A7, online only; Figs $3 \mathrm{~A}$ and $3 \mathrm{~B}$ ). Finally, a post hoc explorative survival analysis showed similar results if only patients with stage IIIC or IV ovarian cancer $(n=140)$ were included (Appendix Table A8, online only; Figs 3C and 3D). No difference in chemotherapy regimen between study groups was seen (Appendix Table A9, online only).

\section{DISCUSSION}

In this multicenter, randomized controlled trial, we investigated the value of laparoscopy in the diagnostic work-up of patients with suspected advanced ovarian cancer. Laparoscopy to guide treatment decision making significantly reduced the percentage of futile PCSs (ie, leaving $>1 \mathrm{~cm}$ of residual disease). Furthermore, by using laparoscopy as a diagnostic aid, the number of patients subjected to multiple laparotomies was significantly reduced without increasing the complication rate.

Cytoreductive surgery with no residual disease $(0 \mathrm{~cm})$ was reached in $41 \%$ of the patients with stage IIIC or IV ovarian cancer who underwent PCS in the laparoscopy group and in only $25 \%$ of the patients in the primary surgery group (Appendix Table A5). These results are comparable to those reported in other multicenter studies that ranged from $17 \%$ to $46 \%$. $^{5,6,8,25-27}$

The strength of this study is the multicenter approach. All eight specialized oncologic centers in the Netherlands participated in this trial, which created an accurate reflection of current practice. The multicenter aspect of the study may be considered an advantage because of its generalizability. On the other hand, different treatment centers and gynecologic oncologists may have differing estimations of the selection of patients considered operable and the chance of futile laparotomies, which could have biased the results. We minimized this effect by establishing clear rules for patient selection and estimations at laparoscopic evaluation.

Because we based our inclusion criteria on conventional work-up and not on histologic biopsy, $13 \%$ of the included patients had either benign or borderline disease or a different origin of malignancy. Furthermore, patients with suspected stage IIB or IIIB ovarian cancer were also included because higher stage or more extensive disease cannot be excluded beforehand, and in these cases, PCS should always be considered. In contrast, the CHORUS 


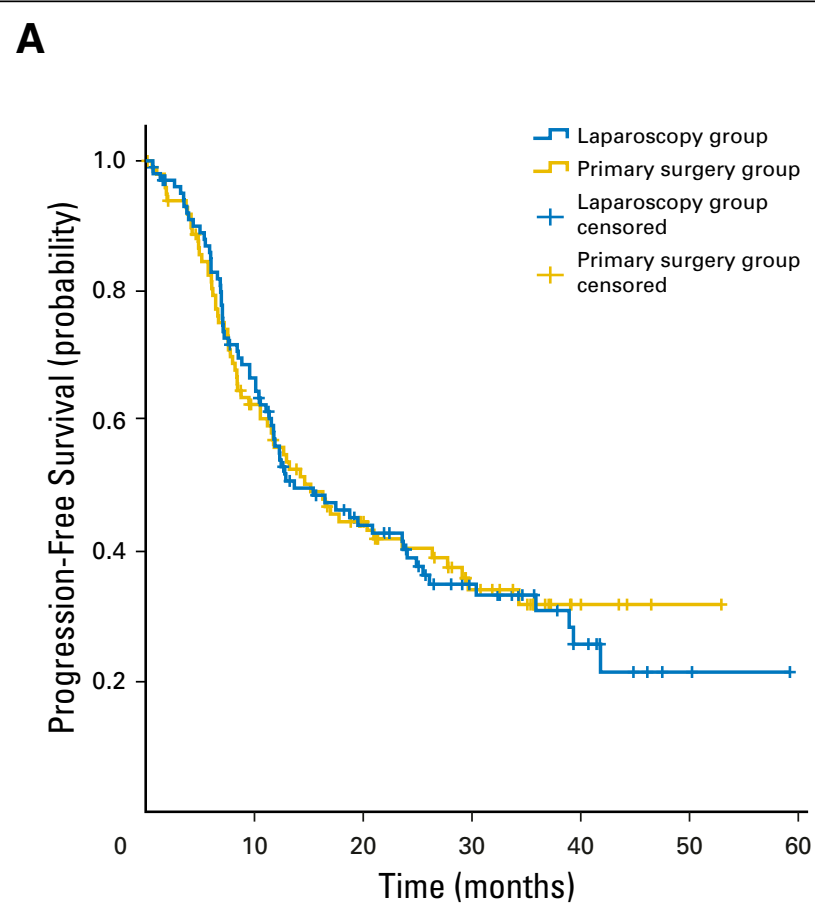

No. at risk:

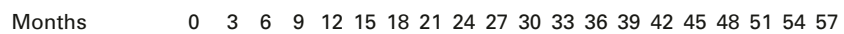

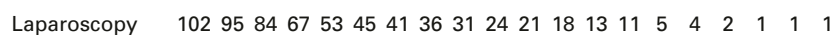
Primary surgery $99 \begin{array}{lllllllllllllllllll}91 & 60 & 50 & 44 & 38 & 32 & 28 & 26 & 19 & 16 & 10 & 7 & 4 & 2 & 1 & 1 & 0 & 0\end{array}$

C

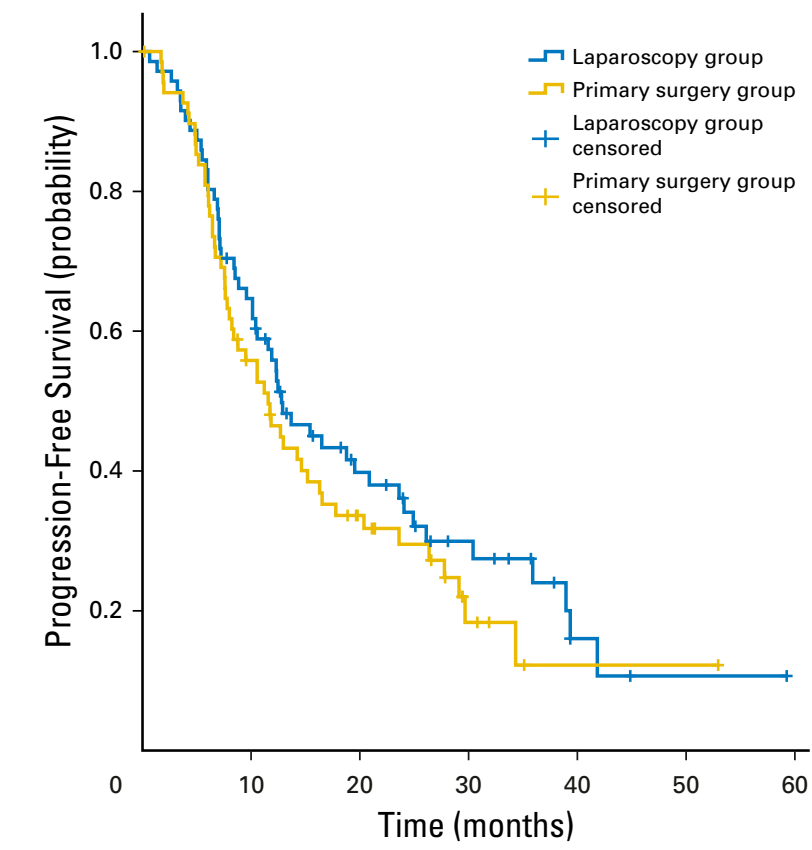

No. at risk:

Months $\quad 0 \quad 3 \quad 6 \quad 9 \quad 12 \quad 15 \quad 182124 \quad 2730 \quad 33 \quad 36 \quad 3942 \quad 4548 \quad 515457$ $\begin{array}{lllllllllllllllllllll}\text { Laparoscopy } & 71 & 68 & 59 & 46 & 37 & 29 & 26 & 21 & 18 & 13 & 12 & 10 & 7 & 5 & 2 & 1 & 1 & 1 & 1 & 1\end{array}$ Primary surgery $69 \begin{array}{lllllllllllllllllllll}55 & 38 & 29 & 25 & 21 & 17 & 13 & 11 & 5 & 3 & 1 & 1 & 1 & 1 & 1 & 1 & 0 & 0\end{array}$
B

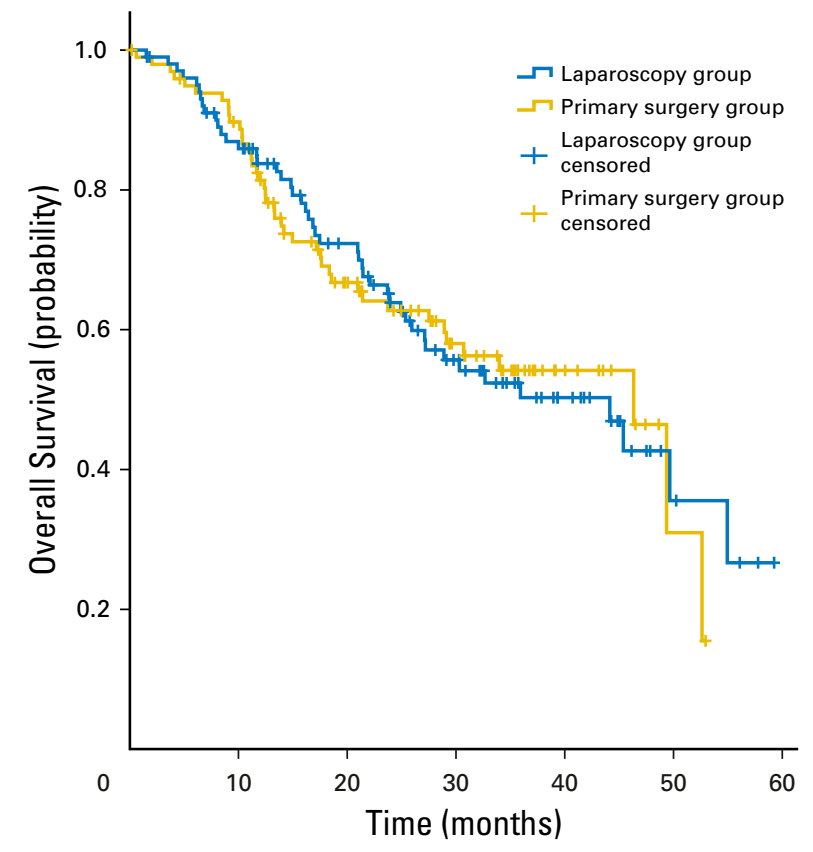

No. at risk:

Months $\quad 0 \quad 3 \quad 6 \quad 9 \quad 12 \quad 151821242730 \quad 3336 \quad 3942 \quad 4548515457$

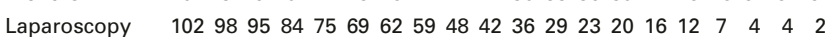

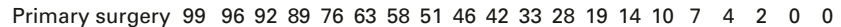

D

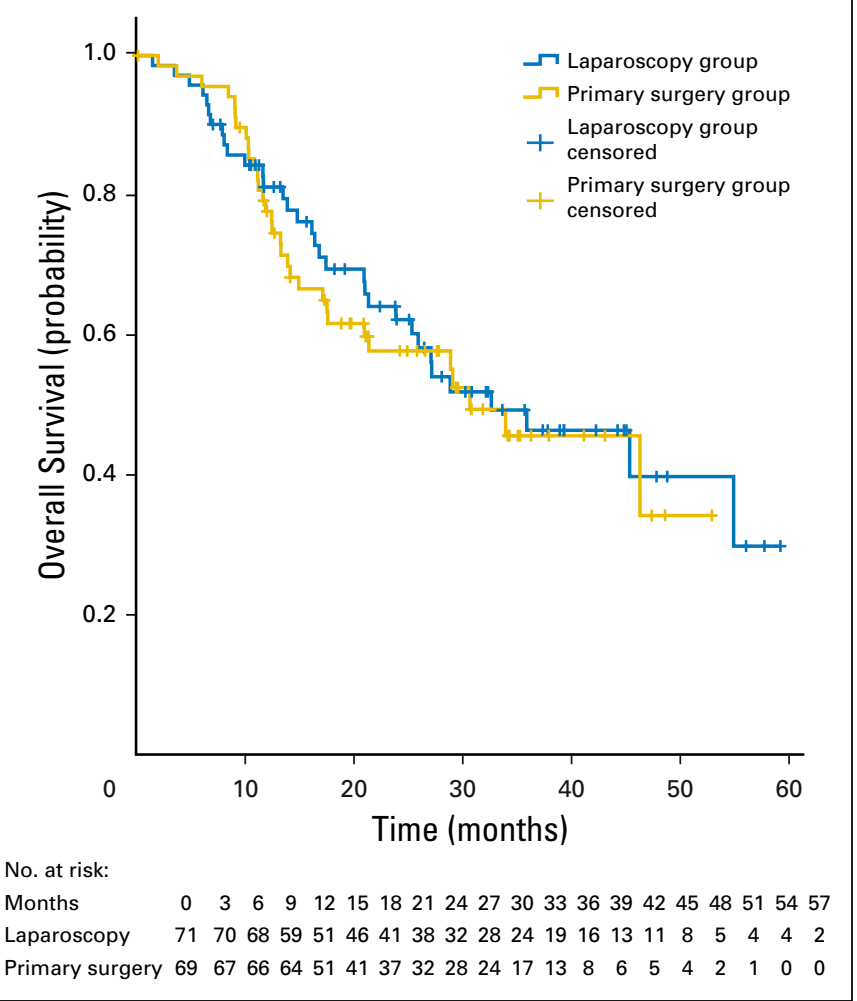

Fig 3. (A) Progression-free survival in intention-to-treat analysis. (B) Overall survival inintention-to-treat analysis. (C) Progression-free survival in subgroup analysis of patients with stage IIIC or IV ovarian cancer. (D) Overall survival in subgroup analysis of patients with stage IIIC or IV ovarian cancer. 
(Primary Chemotherapy Versus Primary Surgery for Newly Diagnosed Advanced Ovarian Cancer) trial used stricter inclusion criteria (ie, included only patients with suspected stage III or IV ovarian cancer) and had 3\% with benign, borderline, or different tumors in their population. ${ }^{5}$ Patients might have been wrongly excluded in the current study in that they may have been considered as having unresectable disease after conventional work-up, which might have influenced the data. Several studies have tried to develop a noninvasive prediction model to predict operability, but none are $100 \%$ accurate. ${ }^{13}$ However, during the inclusion period of the current study, more accurate prediction models for preoperative assessment became available. ${ }^{28-30}$ At the start of our study, however, the Risk of Malignancy Index and CT assessment were part of the standard work-up in the Netherlands.

The reason for exclusion of patients with expected inoperable disease was to prevent patients from being subjected to laparoscopy or laparotomy when primary surgery was considered unfavorable beforehand. Recently, ASCO published a practice guideline that was based on a systematic review that propagated the same approach wherein all women with a high perioperative risk profile and low likelihood of cytoreduction to $<1 \mathrm{~cm}$ are recommended to receive NACT. ${ }^{31}$ PCS is preferred if there is a high likelihood of $<1 \mathrm{~cm}$ of residual disease with acceptable morbidity. Furthermore, four other randomized clinical trials that compared primary surgery and NACT with interval surgery in patients with advanced-stage ovarian cancer concluded that NACT was noninferior and the extent of surgery diminished in the NACT arm with decreased complication rates. ${ }^{5,6,24,32}$ From these results, we conclude that patients with extensive disease, such as large immobile pelvic tumor or extensive disease of the diaphragm, would benefit most from treatment with NACT. ${ }^{31}$ In addition, Vergote et $\mathrm{al}^{6}$ found no survival benefit for either treatment strategy. Therefore, survival was not taken as the primary end point in the current study.

Nevertheless, to our knowledge this randomized clinical study is the first to investigate the role of laparoscopy to prevent futile laparotomies in women with suspected advanced ovarian cancer. Several retrospective or prospective case series have described a diagnostic laparoscopy as a reliable tool to identify women suitable for PCS, but negative predictive values ranged from $69 \%$ to $96 \%$, and heterogeneity of the studies made it impossible to draw firm conclusions. ${ }^{16,33-36}$ A laparoscopy-based score developed in 2005 to 2006 by Fagotti et al ${ }^{14,37}$ showed a positive predictive value of $100 \%$ and a negative predictive value of $70 \%$ for cytoreductive surgery with $<1 \mathrm{~cm}$ of residual disease. This score could not accurately discriminate among women who would be left with $>1 \mathrm{~cm}$ of residual disease after validation. ${ }^{15,38-41}$ Recently, this model was updated by Petrillo et $\mathrm{al}^{33}$ to show a higher discriminating performance of laparoscopy, with an area under the curve of 0.89 and a risk of futile laparotomy of $33 \%$, that leaves any residual disease. Despite these limitations, all the aforementioned studies suggested an additive value of laparoscopy, which was the motivation for the current randomized trial.

In conclusion, if complete PCS seems feasible, diagnostic laparoscopy is an effective and safe tool to select patients in whom PCS will be successful in leaving (at least) $<1 \mathrm{~cm}$ of residual disease. Therefore, diagnostic laparoscopy should be considered in the diagnostic work-up of women with ovarian cancer to guide treatment selection for either PCS or NACT.

\section{AUTHORS' DISCLOSURES OF POTENTIAL CONFLICTS OF INTEREST}

Disclosures provided by the authors are available with this article at ascopubs.org/journal/jco.

\section{AUTHOR CONTRIBUTIONS}

Conception and design: Marianne J. Rutten, Hannah S. van Meurs, Toon van Gorp, Henk G. Ter Brugge, Ward Hofhuis, Henk W.R. Schreuder, Henriette J.G. Arts, Petra L.M. Zusterzeel, Johanna M.A. Pijnenborg, Maarten van Haaften, Mirjam J.A. Engelen, Erik A. Boss, M. Caroline Vos, Eltjo M.J. Schutter, Brent C. Opmeer, Anje M. Spijkerboer, Ben Willem Mol, Gemma G. Kenter, Marrije R. Buist

Collection and assembly of data: Marianne J. Rutten, Hannah S. van Meurs, Roelien van de Vrie, Katja N. Gaarenstroom, Toon van Gorp, Ward Hofhuis, Henriette J.G. Arts, Petra L.M. Zusterzeel, Johanna M.A. Pijnenborg, Maarten van Haaften, Guus Fons, Mirjam J.A. Engelen, Kees G. Gerestein, Anje M. Spijkerboer, Patrick M.M. Bossuyt, Ben Willem Mol, Gemma G. Kenter, Marrije R. Buist

Data analysis and interpretation: Marianne J. Rutten, Hannah S. van Meurs, Roelien van de Vrie, Katja N. Gaarenstroom, Christiana A. Naaktgeboren, Toon van Gorp, Henk G. Ter Brugge, Ward Hofhuis, Henk W.R. Schreuder, Henriette J.G. Arts, Petra L.M. Zusterzeel, Johanna M.A. Pijnenborg, Maarten van Haaften, Mirjam J.A. Engelen, Erik A. Boss, M. Caroline Vos, Eltjo M.J. Schutter, Anje M. Spijkerboer, Patrick M.M. Bossuyt, Ben Willem Mol, Gemma G. Kenter, Marrije R. Buist

Manuscript writing: All authors

Final approval of manuscript: All authors

Accountable for all aspects of the work: All authors

\section{REFERENCES}

1. Ferlay $J$, Soerjomatraram I, Ervik $M$, et al: Globocan 2012 v1.1, Cancer Incidence and Mortality Worldwide: IARC CancerBase No. 11. Lyon, France, International Agency for Research on Cancer, 2013

2. Rose $P G$, Nerenstone $S$, Brady MF, et al: Secondary surgical cytoreduction for advanced ovarian carcinoma. N Engl J Med 351:2489-2497, 2004

3. van der Burg ME, van Lent $M$, Buyse $M$, et al: The effect of debulking surgery after induction chemotherapy on the prognosis in advanced epithelial ovarian cancer. N Engl J Med 332:629-634, 1995
4. Makar AP, Tropé CG, Tummers $P$, et al: Advanced ovarian cancer: Primary or interval debulking? Five categories of patients in view of the results of randomized trials and tumor biology: Primary debulking surgery and interval debulking surgery for advanced ovarian cancer. Oncologist 21:745-754, 2016

5. Kehoe S, Hook J, Nankivell M, et al: Primary chemotherapy versus primary surgery for newly diagnosed advanced ovarian cancer (CHORUS): An open-label, randomised, controlled, non-inferiority trial. Lancet 386:249-257, 2015

6. Vergote I, Tropé CG, Amant F, et al: Neoadjuvant chemotherapy or primary surgery in stage IIIC or IV ovarian cancer. N Engl J Med 363:943-953, 2010
7. van Meurs HS, Tajik $\mathrm{P}$, Hof $\mathrm{MH}$, et al: Which patients benefit most from primary surgery or neoadjuvant chemotherapy in stage IIIC or IV ovarian cancer? An exploratory analysis of the European Organisation for Research and Treatment of Cancer 55971 randomised trial. Eur J Cancer 49:3191-3201, 2013

8. Mueller JJ, Zhou QC, lasonos A, et al: Neoadjuvant chemotherapy and primary debulking surgery utilization for advanced-stage ovarian cancer at a comprehensive cancer center. Gynecol Oncol 140: 436-442, 2016

9. Bristow RE: Predicting surgical outcome for advanced ovarian cancer, surgical standards of care, and the concept of kaizen. Gynecol Oncol 112:1-3, 2009 
10. Gerestein CG, Nieuwenhuyzen-de Boer GM, Eijkemans MJ, et al: Prediction of 30-day morbidity after primary cytoreductive surgery for advanced stage ovarian cancer. Eur J Cancer 46:102-109, 2010

11. Aletti GD, Dowdy SC, Podratz KC, et al: Relationship among surgical complexity, short-term morbidity, and overall survival in primary surgery for advanced ovarian cancer. Am J Obstet Gynecol 197:676.e1-676.e7, 2007

12. Vergote I, van Gorp T, Amant $F$, et al: Timing of debulking surgery in advanced ovarian cancer. Int $\mathrm{J}$ Gynecol Cancer 18:11-19, 2008 (suppl 1)

13. Rutten MJ, van de Vrie $R$, Bruining $A$, et al: Predicting surgical outcome in patients with International Federation of Gynecology and Obstetrics stage III or IV ovarian cancer using computed tomography: A systematic review of prediction models. Int J Gynecol Cancer 25:407-415, 2015

14. Fagotti $A$, Fanfani $F$, Ludovisi $M$, et al: Role of laparoscopy to assess the chance of optimal cytoreductive surgery in advanced ovarian cancer: A pilot study. Gynecol Oncol 96:729-735, 2005

15. Fagotti A, Ferrandina G, Fanfani F, et al: Prospective validation of a laparoscopic predictive model for optimal cytoreduction in advanced ovarian carcinoma. Am J Obstet Gynecol 199:642.e1-642.e6, 2008

16. Rutten MJ, Leeflang MM, Kenter GG, et al: Laparoscopy for diagnosing resectability of disease in patients with advanced ovarian cancer. Cochrane Database Syst Rev 2:CD009786, 2014

17. Pecorelli S, Benedet JL, Creasman WT, et al: FIGO staging of gynecologic cancer. 1994-1997 FIGO Committee on Gynecologic Oncology. Int J Gynaecol Obstet 65:243-249, 1999

18. Jacobs I, Oram D, Fairbanks J, et al: A risk of malignancy index incorporating CA 125, ultrasound and menopausal status for the accurate preoperative diagnosis of ovarian cancer. Br J Obstet Gynaecol 97: 922-929, 1990

19. Engelen MJA, Kos HE, Willemse PHB, et al: Surgery by consultant gynecologic oncologists improves survival in patients with ovarian carcinoma. Cancer 106:589-598, 2006

20. Rutten MJ, Gaarenstroom KN, Van Gorp T, et al: Laparoscopy to predict the result of primary cytoreductive surgery in advanced ovarian cancer patients (LapOvCa-trial): A multicentre randomized controlled study. BMC Cancer 12:31, 2012
21. Silverberg SG: Histopathologic grading of ovarian carcinoma: A review and proposal. Int J Gynecol Pathol 19:7-15, 2000

22. Vergote I, Marquette S, Amant F, et al: Portsite metastases after open laparoscopy: A study in 173 patients with advanced ovarian carcinoma. Int J Gynecol Cancer 15:776-779, 2005

23. Kruitwagen RF, Swinkels BM, Keyser KG, et al: Incidence and effect on survival of abdominal wall metastases at trocar or puncture sites following laparoscopy or paracentesis in women with ovarian cancer. Gynecol Oncol 60:233-237, 1996

24. Heitz F, Ognjenovic D, Harter P, et al: Abdominal wall metastases in patients with ovarian cancer after laparoscopic surgery: Incidence, risk factors, and complications. Int J Gynecol Cancer 20:41-46, 2010

25. Fagotti A, Ferrandina G, Vizzielli G, et al: Phase III randomised clinical trial comparing primary surgery versus neoadjuvant chemotherapy in advanced epithelial ovarian cancer with high tumour load (SCORPION trial): Final analysis of peri-operative outcome. Eur J Cancer 59:22-33, 2016

26. Chi DS, Zivanovic O, Levinson $K L$, et al: The incidence of major complications after the performance of extensive upper abdominal surgical procedures during primary cytoreduction of advanced ovarian, tubal, and peritoneal carcinomas. Gynecol Oncol 119:38-42, 2010

27. Barlin JN, Yu C, Hill EK, et al: Nomogram for predicting 5-year disease-specific mortality after primary surgery for epithelial ovarian cancer. Gynecol Oncol 125:25-30, 2012

28. Kaijser J, Sayasneh A, Van Hoorde $K$, et al: Presurgical diagnosis of adnexal tumours using mathematical models and scoring systems: A systematic review and meta-analysis. Hum Reprod Update 20:449-462, 2014

29. Van Calster B, Van Hoorde K, Valentin L, et al: Evaluating the risk of ovarian cancer before surgery using the ADNEX model to differentiate between benign, borderline, early and advanced stage invasive, and secondary metastatic tumours: Prospective multicentre diagnostic study. BMJ 349 : g5920, 2014

30. Szubert S, Wojtowicz A, Moszynski R, et al: External validation of the IOTA ADNEX model performed by two independent gynecologic centers. Gynecol Oncol 142:490-495, 2016

31. Wright AA, Bohlke $K$, Armstrong DK, et al: Neoadjuvant chemotherapy for newly diagnosed, advanced ovarian cancer: Society of Gynecologic Oncology and American Society of Clinical Oncology clinical practice guideline. J Clin Oncol 34:3460-3473, 2016

32. Onda $T$, Satoh $T$, Saito $T$, et al: Comparison of treatment invasiveness between upfront debulking surgery versus interval debulking surgery following neoadjuvant chemotherapy for stage III/IV ovarian, tubal, and peritoneal cancers in a phase III randomised trial: Japan Clinical Oncology Group Study JCOG0602. Eur J Cancer 64:22-31, 2016

33. Petrillo M, Vizzielli G, Fanfani F, et al: Definition of a dynamic laparoscopic model for the prediction of incomplete cytoreduction in advanced epithelial ovarian cancer: Proof of a concept. Gynecol Oncol 139:5-9, 2015

34. Fanfani F, Monterossi G, Fagotti A, et al: Positron emission tomography-laparoscopy based method in the prediction of complete cytoreduction in platinum-sensitive recurrent ovarian cancer. Ann Surg Oncol 22:649-654, 2015

35. Brun JL, Rouzier R, Selle F, et al: Neoadjuvant chemotherapy or primary surgery for stage III/IV ovarian cancer: Contribution of diagnostic laparoscopy. BMC Cancer 9:171, 2009

36. Angioli R, Palaia I, Zullo MA, et al: Diagnostic open laparoscopy in the management of advanced ovarian cancer. Gynecol Oncol 100:455-461, 2006

37. Fagotti A, Ferrandina G, Fanfani F, et al: A laparoscopy-based score to predict surgical outcome in patients with advanced ovarian carcinoma: A pilot study. Ann Surg Oncol 13:1156-1161, 2006

38. Fagotti A, Fanfani F, Vizzielli G, et al: Should laparoscopy be included in the work-up of advanced ovarian cancer patients attempting interval debulking surgery? Gynecol Oncol 116:72-77, 2010

39. Varnoux C, Huchon C, Bats AS, et al: Diagnostic accuracy of hand-assisted laparoscopy in predicting resectability of peritoneal carcinomatosis from gynecological malignancies. Eur J Surg Oncol 39:774-779, 2013

40. Chereau E, Lavoue V, Ballester M, et al: External validation of a laparoscopic-based score to evaluate resectability for patients with advanced ovarian cancer undergoing interval debulking surgery. Anticancer Res 31:4469-4474, 2011

41. Ramirez PT: Upping the ante in assessing the feasibility of surgical cytoreduction for advanced ovarian cancer: A modified laparoscopic predictive index score. Gynecol Oncol 139:1-2, 2015

\section{Affiliations}

Marianne J. Rutten, Hannah S. van Meurs, Roelien van de Vrie, Christiana A. Naaktgeboren, Guus Fons, Brent C. Opmeer, Anje M. Spijkerboer, Patrick M.M. Bossuyt, Gemma G. Kenter, and Marrije R. Buist, Academic Medical Center, Amsterdam; Katja N. Gaarenstroom, Leiden University Medical Center, Leiden; Toon van Gorp, Maastricht University Medical Center, Maastricht; Henk G. Ter Brugge, Isala Hospital, Zwolle; Ward Hofhuis, Sint Franciscus Gasthuis, Rotterdam; Henk W.R. Schreuder, University Medical Center Utrecht; Maarten van Haaften, Diakonessenhuis, Utrecht; Henriette J.G. Arts, University Medical Center Groningen, Groningen; Petra L.M. Zusterzeel, Radboud University Medical Center, Nijmegen; Johanna M.A. Pijnenborg and M. Caroline Vos, Elisabeth-Tweesteden Hospital, Tilburg; Mirjam J.A. Engelen, Atrium Medical Center, Heerlen; Erik A. Boss, Máxima Medical Center, Veldhoven; Kees G. Gerestein, Meander Medical Center, Amersfoort; Eltjo M.J. Schutter, Medical Spectrum Twente, Enschede, the Netherlands; and Ben Willem Mol, The Robinson Research Institute, University of Adelaide; The South Australian Health and Medical Research Institute, Adelaide, Australia.

\section{Support}

Supported by the Dutch Organization for Health Research and Development (project number 171102021). The funder of the study had no role in the study design, data collection, data analysis, data interpretation, or writing of the manuscript.

\section{Prior Presentation}

Presented at the International Meeting of the European Society of Gynaecological Oncology, Nice, France, October 24-27, 2015. 
Laparoscopy to Predict the Result of Primary Cytoreductive Surgery in Patients With Advanced Ovarian Cancer: A Randomized Controlled Trial

The following represents disclosure information provided by authors of this manuscript. All relationships are considered compensated. Relationships are self-held unless noted. I = Immediate Family Member, Inst = My Institution. Relationships may not relate to the subject matter of this manuscript. For more information about ASCO's conflict of interest policy, please refer to www.asco.org/rwc or ascopubs.org/jco/site/ifc.

Marianne J. Rutten

No relationship to disclose

Hannah S. van Meurs

No relationship to disclose

Roelien van de Vrie

No relationship to disclose

Katja N. Gaarenstroom

No relationship to disclose

Christiana A. Naaktgeboren

No relationship to disclose

Toon van Gorp

No relationship to disclose

Henk G. Ter Brugge

No relationship to disclose

Ward Hofhuis

No relationship to disclose

Henk W.R. Schreuder

No relationship to disclose

Henriette J.G. Arts

No relationship to disclose

Petra L.M. Zusterzeel

No relationship to disclose

Johanna M.A. Pijnenborg

No relationship to disclose

Maarten van Haaften

No relationship to disclose

Guus Fons

No relationship to disclose

\section{Mirjam J.A. Engelen}

No relationship to disclose

Erik A. Boss

No relationship to disclose

M. Caroline Vos

No relationship to disclose

Kees G. Gerestein

No relationship to disclose

Eltjo M.J. Schutter

No relationship to disclose

Brent C. Opmeer

No relationship to disclose

Anje M. Spijkerboer

No relationship to disclose

Patrick M.M. Bossuyt

No relationship to disclose

Ben Willem Mol

Honoraria: ObsEva

Consulting or Advisory Role: ObsEva

Research Funding: National Health and Medical Research Council (Inst), Human Tissue Authority UK (Inst), Dutch Organization for Health Research and Development (Inst)

Travel, Accommodations, Expenses: European Society of Human Reproduction and Embryology, Society for Maternal-Fetal Medicine, International Society for the Study of Hypertension in Pregnancy

Gemma G. Kenter

No relationship to disclose

\section{Marrije R. Buist}

No relationship to disclose 


\section{Acknowledgment}

We thank Professor Dr Ignace Vergote for his role as project advisor. We thank all the women who participated in this trial, all investigators and supporters at the study sites, the central study office of the study groups, and all involved staff at Academic Medical Center of Amsterdam. Special thanks to Fon M. Kosterman, research nurse at Academic Medical Center of Amsterdam, and to all including gynecologists and their research staff; Dr Johannes van der Velden of Academic Medical Center, Amsterdam; Dr Hans Trum of VU University Medical Center of Amsterdam; Dr Willemien van Driel of Antoni van Leeuwenhoek Hospital, Amsterdam; Dr Marchien van Baal of Flevoziekenhuis, Almere; Dr Harold R. Verhoeve of Onze Lieve Vrouwen Gasthuis, Amsterdam; Dr Annechien Bouman of Deventer Hospital, Deventer; Dr Joke Klinkert of Westfriesgasthuis, Hoorn; Dr Marcel Huisman of Gelre Hospital, Apeldoorn; Dr John Dawson of Sint Lucas Andreas Hospital, Amsterdam; Dr Thomas K.G.Tavernier of Gemini Hospital, Den Helder; Dr Celesta Wensveen of Erasmus Medical Center, Rotterdam; Dr Arjan Kraayenbrink of Rijnstate, Arnhem; and Dr Jules .H. Schagen van Leeuwen of Antonius Hospital, Nieuwegein, the Netherlands.

\section{Appendix}

\begin{tabular}{|c|c|c|c|c|}
\hline Outcome & Laparoscopy Before Surgery $(n=101)$, No. $(\%)$ & Primary Surgery $(n=94)$, No. $(\%)$ & Relative Risk (95\% Cl) & $P$ \\
\hline Futile laparotomy* & $10(10)$ & $39(42)$ & 0.24 (0.13 to 0.45$)$ & $<.001$ \\
\hline Futile laparotomy any residue $\dagger$ & $27(27)$ & $56(65)$ & 0.45 (0.31 to 0.65$)$ & $<.001$ \\
\hline
\end{tabular}

Table A2. Characteristics of Treatment, Extent of Surgery, and Response After Completion of Treatment, Including Chemotherapy Per Treatment Arm

\begin{tabular}{|c|c|c|c|c|}
\hline Characteristic & Laparoscopy Before Surgery, No. (\%) & Primary Surgery, No. (\%) & $\mathrm{RR}(95 \% \mathrm{Cl})$ & $P$ \\
\hline \multicolumn{5}{|l|}{ Extent of first cytoreductive surgery* } \\
\hline No. of patients & 98 & 98 & & \\
\hline Extensive surgery performed $\dagger$ & $35(36)$ & $26(27)$ & $1.35(0.88$ to 2.06$)$ & .22 \\
\hline Hysterectomy & $80(82) \ddagger$ & $60(61) \S$ & $1.32(1.11$ to 1.60$)$ & .002 \\
\hline Bilateral salpingo-oophorectomy & $89(92)$ & $70(71)$ & $1.29(1.12$ to 1.48$)$ & $<.001$ \\
\hline Omentectomy & $82(84)$ & $60(61)$ & 1.37 (1.14 to 1.64$)$ & $<.001$ \\
\hline Pelvic lymphadenectomy & $7(7)$ & $10(10)$ & $0.70(0.28$ to 1.76$)$ & .45 \\
\hline Para-aortic lymphadenectomy & $5(5)$ & $8(8)$ & $0.63(0.21$ to 1.86$)$ & .40 \\
\hline Peritoneal stripping & $26(26)$ & $20(20)$ & $1.30(0.78$ to 1.17$)$ & .31 \\
\hline Small bowel resection & $6(6)$ & $3(3)$ & $2.00(0.52$ to 7.77$)$ & .50 \\
\hline Large bowel resection & $13(13)$ & $10(10)$ & $1.30(0.60$ to 2.82$)$ & .51 \\
\hline Ostomy & 4 (4) & $1(1)$ & $4.00(0.46$ to 35.2$)$ & .37 \\
\hline Diaphragm stripping & $16(16)$ & $9(9)$ & $1.78(0.83$ to 3.83$)$ & .13 \\
\hline Splenectomy & $2(2)$ & $2(2)$ & $1.01(0.15$ to 7.03$)$ & 1.00 \\
\hline (Partial) liver resection & $1(1)$ & $1(1)$ & $1.00(0.06$ to 15.76$)$ & 1.00 \\
\hline Response after completion of treatment\| & 99 & 95 & & \\
\hline Complete remission & $65(66)$ & $65(68)$ & & .39 \\
\hline Partial remission & 9 (9) & $14(15)$ & & \\
\hline Stable disease & $7(7)$ & $5(5)$ & & \\
\hline Progressive disease & $18(18)$ & $11(12)$ & & \\
\hline
\end{tabular}

Abbreviation: $\mathrm{RR}$, relative risk.

* Five patients did not receive cytoreductive surgery.

tExtensive surgery consisted of at least one of the following procedures: peritoneal stripping, small or large bowel resection, ostomy construction, diaphragm stripping, splenectomy, or (partial) liver resection.

fThirteen of these 80 patients had a history of hysterectomy; 11 had no hysterectomy as a result of futile laparotomy with $>1 \mathrm{~cm}$ of residual disease; one had no hysterectomy because of a different malignancy; four had benign disease; two had no malignant aspect of the uterus and therefore no hysterectomy.

$\S$ Fourteen of these 60 patients had a history of hysterectomy; thirty had no hysterectomy as a result of futile laparotomy with $>1 \mathrm{~cm}$ of residual disease; four had benign or borderline disease; two had International Federation of Gynecology and Obstetrics stage I ovarian cancer. For one patient, the uterus was not removed because of a primary bladder tumor and adhesions of uterus and bladder; one patient had extensive peritonitis; therefore, no hysterectomy was performed, with residual disease of $<1 \mathrm{~cm}$.

\|Response after completion of treatment was assessed 6 weeks after the last cycle of chemotherapy; data are missing for seven patients. 


\begin{tabular}{|c|c|c|c|c|}
\hline Characteristic & Laparoscopy Before Debulking, No. (\%) & Primary Debulking, No. (\%) & $\operatorname{RR}(95 \% \mathrm{Cl})$ & $P$ \\
\hline \multicolumn{5}{|l|}{ Extent of first cytoreductive surgery* } \\
\hline No. of patients & 68 & 68 & & \\
\hline Extensive surgery performed $\dagger$ & $29(43)$ & $21(31)$ & 1.38 (0.88 to 2.17$)$ & .16 \\
\hline Hysterectomy & $58(85) \ddagger$ & $41(60) \S$ & $1.42(1.14$ to 1.76$)$ & .001 \\
\hline Bilateral salpingo-oophorectomy & $61(90)$ & $44(65)$ & $1.41(1.16$ to 1.70$)$ & $<.001$ \\
\hline Omentectomy & $61(90)$ & $43(63)$ & $1.42(1.16$ to 1.73$)$ & $<.001$ \\
\hline Pelvic lymphadenectomy & $3(4)$ & $4(6)$ & $0.75(0.17$ to 3.23$)$ & .70 \\
\hline Para-aortic lymphadenectomy & 2 (3) & $3(4)$ & $0.68(0.12$ to 3.92$)$ & .66 \\
\hline Peritoneal stripping & $22(32)$ & $17(25)$ & $1.29(0.76$ to 2.21$)$ & .34 \\
\hline Small bowel resection & $6(9)$ & $3(4)$ & $2.00(0.52$ to 7.67$)$ & .30 \\
\hline Large bowel resection & $11(16)$ & $8(12)$ & $1.38(0.59$ to 3.21$)$ & .46 \\
\hline Ostomy & $3(4)$ & $1(2)$ & $3.00(0.32$ to 28.13$)$ & .31 \\
\hline Diaphragm stripping & $14(21)$ & $8(12)$ & $1.75(0.79$ to 3.90$)$ & .16 \\
\hline Splenectomy & $1(2)$ & $2(3)$ & $0.51(0.05$ to 5.47$)$ & .57 \\
\hline (Partial) liver resection & $1(2)$ & $1(2)$ & $1.00(0.06$ to 15.66$)$ & 1.00 \\
\hline Response after completion of treatment|| & 70 & 67 & & \\
\hline Complete remission & $45(64)$ & $41(61)$ & & .46 \\
\hline Partial remission & $8(11)$ & $13(19)$ & & \\
\hline Stable disease & $4(6)$ & $5(8)$ & & \\
\hline Progressive disease & $13(19)$ & $8(12)$ & & \\
\hline
\end{tabular}

Abbreviation: $\mathrm{RR}$, relative risk.

* Five patients did not receive cytoreductive surgery.

tExtensive surgery consisted of at least one of the following procedures: peritoneal stripping, small or large bowel resection, ostomy construction, diaphragm stripping, splenectomy, or (partial) liver resection.

¥Ten of these 58 patients had a history of hysterectomy; eight had no hysterectomy during the first surgery as a result of futile laparotomy with > 1 cm of residual disease; two had no malignant aspect of the uterus and therefore no hysterectomy; three had no cytoreductive surgery.

$\S$ Seven of these 41 patients had a history of hysterectomy, and 25 had no hysterectomy as a result of futile laparotomy with $>1 \mathrm{~cm}$ of residual disease. For one patient, the uterus was not removed because of a primary bladder tumor and adhesions of uterus and bladder, and one patient had extensive peritonitis; therefore, no hysterectomy was performed, with residual disease of $<1 \mathrm{~cm}$.

$\|$ Response after completion of treatment was assessed 6 weeks after the last cycle of chemotherapy; data missing for three patients.

Table A4. Outcome of First Cytoreductive Surgery Per Treatment Arm for All Patients, Specified Per Type of Surgery: Primary Cytoreductive Surgery or Interval Cytoreductive Surgery

\begin{tabular}{|c|c|c|c|}
\hline Type of First Surgery and Outcome & Laparoscopy Before Surgery, No. (\%) & Primary Surgery, No. (\%) & $P$ \\
\hline No. of patients & 102 & 99 & \\
\hline \multicolumn{4}{|l|}{ Primary cytoreductive surgery } \\
\hline No. of patients & $63(62)$ & 93 (94) & \\
\hline No residual disease & $36(57)$ & $37(40)$ & .003 \\
\hline $0.1-1 \mathrm{~cm}$ of residual disease & $17(27)$ & $17(18)$ & \\
\hline$>1 \mathrm{~cm}$ of residual disease & $10(16)$ & $39(42)$ & \\
\hline \multicolumn{4}{|l|}{ Interval cytoreductive surgery } \\
\hline No. of patients & 35 (34) & 5 (5) & \\
\hline No residual disease & $18(51)$ & $3(60)$ & .94 \\
\hline $0.1-1 \mathrm{~cm}$ of residual disease & $9(26)$ & $1(20)$ & \\
\hline$>1 \mathrm{~cm}$ of residual disease & $8(23)$ & $1(20)$ & \\
\hline
\end{tabular}


Table A5. Subgroup Analysis: Outcome of First Cytoreductive Surgery Per Treatment Arm for Patients With Confirmed Stage IIIC or IV Ovarian Cancer

\begin{tabular}{|c|c|c|c|}
\hline Type of First Surgery and Outcome* & Laparoscopy Before Surgery, No. (\%) & Primary Surgery, No. (\%) & $P$ \\
\hline No. of patients & 68 & 68 & \\
\hline \multicolumn{4}{|l|}{ Primary cytoreductive surgery } \\
\hline No. of patients & 34 & 63 & \\
\hline $0.1-1 \mathrm{~cm}$ of residual disease & $14(41)$ & $15(24)$ & \\
\hline$>1 \mathrm{~cm}$ of residual disease & $6(18)$ & $32(51)$ & \\
\hline \multicolumn{4}{|l|}{ Interval cytoreductive surgery } \\
\hline No. of patients & 34 & 5 & \\
\hline$>1 \mathrm{~cm}$ of residual disease & $7(21)$ & $1(20)$ & \\
\hline
\end{tabular}

NOTE. Outcome of first cytoreductive surgery per treatment arm, specified per type of surgery: primary cytoreductive surgery or interval cytoreductive surgery.

*Five patients did not receive cytoreductive surgery.

\begin{tabular}{|c|c|c|c|c|}
\hline No. of patients & 102 & 99 & & \\
\hline Grade 3 or 4 complication per arm & $28(27)$ & $38(38)$ & & 38 \\
\hline Postsurgical complications with surgical intervention & $3(3)$ & $4(4)$ & & \\
\hline Postoperative infections & $4(4)$ & $6(6)$ & & \\
\hline Blood transfusions & $6(6)$ & $4(4)$ & & \\
\hline Thrombosis/embolism & $3(3)$ & $3(3)$ & & \\
\hline Other & $4(4)$ & 7 (7) & & \\
\hline Grade 3 or 4 complications related to laparoscopy & $1(1)^{*}$ & NA & & \\
\hline Death $<28$ days after surgery & 0 & $1(1) \dagger$ & & \\
\hline Port-site metastases & $3(3)$ & NA & & \\
\hline Death related to malignancy & $45(44)$ & 37 (37) & $1.11(0.99$ to 1.25$)$ & .07 \\
\hline
\end{tabular}

\begin{tabular}{|c|c|c|c|c|}
\hline Characteristic & Laparoscopy Before Debulking, Median (IQR) & Primary Debulking, Median (IQR) & HR $(95 \%$ Cl) & $P$ \\
\hline No. of patients & 102 & 99 & & \\
\hline Progression-free survival, months & 13.7 (7.1 to 41.8$)$ & $15.2(7.2$ to $>34)$ & 0.97 (0.69 to 1.38$)$ & .88 \\
\hline Overall survival, months & 44.4 (16.8 to > 55) & 46.3 (13.9 to 52.6$)$ & $1.08(0.76$ to 1.54$)$ & .94 \\
\hline Duration of follow-up, months & 34.6 (24.0 to 45.1$)$ & 33.8 (21.4 to 39.0$)$ & & 16 \\
\hline
\end{tabular}


Rutten et al

Table A8. Subgroup Survival Analysis of Patients With Stage IIIC or IV Ovarian Cancer: Progression-Free Survival and Overall Survival Per Treatment Arm

Characteristic

No. of patients

Progression-free survival, months

Overall survival, months

Duration of follow-up, months
Laparoscopy Before Debulking, Median (IQR) 71

12.8 (7.1 to 35.9$)$

$32.7(16.2$ to $>55)$

35.7 (23.8 to 44.8 )
Primary Debulking, Median (IQR)

69

$11.6(6.4$ to 29.7$)$

$30.7(12.5$ to $>46)$

39.4 (21.3 to 36.3 )
HR $(95 \% \mathrm{Cl})$

1.22 (0.83 to 1.81$)$

$1.33(0.89$ to 1.98$)$ .32 .73

Abbreviations: $\mathrm{HR}$, hazard ratio; IQR, interquartile range.

Table A9. Chemotherapy Received Per Treatment Arm of the Entire Study Group

\begin{tabular}{|c|c|c|c|c|}
\hline Chemotherapy & Laparoscopy Before Surgery, No. (\%) & Primary Surgery, No. (\%) & $\operatorname{RR}(95 \% \mathrm{Cl})$ & $P$ \\
\hline No. of patients & 101 & 94 & & \\
\hline Patients who received chemotherapy* & $87(86)$ & $85(87)$ & 0.99 (0.89 to 1.11$)$ & 1.0 \\
\hline \multicolumn{5}{|l|}{ Treatment } \\
\hline Taxane/platinum regimen & $73(82)$ & $71(86)$ & & .53 \\
\hline Other & $16(18)$ & $12(15)$ & & \\
\hline Completed six courses & $66(66)$ & $70(73)$ & $0.91(0.75$ to 1.09$)$ & .29 \\
\hline
\end{tabular}

Abbreviation: $R R$, relative risk.

* Standard chemotherapy doses were paclitaxel $175 \mathrm{mg} / \mathrm{m}^{2}$ and carboplatin with an area under the curve of 6 on the basis of creatinine clearance every 3 weeks for six courses. 